\title{
REVISIÓN DE TEMA: PAPEL DE LA ACTIVIDAD FÍSICA EN EL MANEJO NO FARMACOLÓGICO DEL SÍNDROME DE IN- TESTINO IRRITABLE
}

\section{ROLE OF PHYSICAL ACTIVITY IN THE NON PHARMACOLOGIC TREATMENT OF IRRITABLE BOWEL SYNDROME: A LI- TERATURE REVIEW}

Pág. 36,41

Recibido: $30-03-2019$

Aceptado: 12-05-2020

Dra. Mariana Villalobos-Jiménez: Médico General. San José, Costa Rica.

Dr. Yoel Merenstein-Hoffman: Médico General. San José, Costa Rica.

Dr. Fabian Rodriguez Palma: Médico General. Caja Costarricense de Seguro Social. San José, Costa Rica.

Dra. Camila Castro-Durán: Médico General. San José, Costa Rica.

Dr. Ricardo Camacho Morales: Médico General. Universidad de Costa Rica. San José, Costa Rica.

\section{Financiamiento}

La presente investigación no ha recibido ayudas específicas provenientes de agencias del sector público, sector comercial o entidades sin ánimo de lucro.

\section{Resumen}

El síndrome de intestino irritable (SII) es el diagnóstico gastroenterológico más común, con una elevada prevalencia global que oscila entre el $5 \%$ al $21 \%$. Esta enfermedad de curso recurrente crónico se caracteriza por su traslape con otros trastornos funcionales y su afectación en la calidad de vida de los pacientes. Actualmente son pocos los tratamientos que ofrecen un alivio sintomático satisfactorio, por lo que en el manejo efectivo del SIl se ha dado importancia a la consejería sobre cambios en el estilo de vida, actividad física, dieta, entre otros. Este enfoque de esfuerzo personal por parte del paciente se sustenta en la relación del estado de estrés crónico con la exacerbación de los síntomas de SII. Las interacciones cerebro-intestino pueden jugar un rol en la asociación entre la actividad física y los síntomas en el SII. La mayoría de estudios concuerdan que las intervenciones que propician el aumento de la actividad física mejoran los síntomas gastrointestinales del SII; así como diferentes aspectos de la calidad de vida, fatiga, depresión y ansiedad a largo plazo.

Palabras claves: Síndrome de intestino irritable, enfermedades gastrointestinales, ejercicio, actividad física, revisión.

\section{Abstract}

Irritable bowel syndrome (IBS) is the most common gastroenterological diagnosis, with a high overall prevalence ranging from $5 \%$ to $21 \%$. This chronic recurrent disease course is characterized by its overlap with other functional disorders and its impact on quality of life of the patients. Currently there are few treatments that offer satisfactory symptomatic relief, so in the effective management of IBS importance has been given to counseling on changes in lifestyle, physical activity, diet, among others. This approach to personal effort by the patient is based on the relationship of the chronic stress state with the exacerbation of IBS symptoms. Brain-gut interactions may play a role in the association between physical activity and symptoms in IBS. Most studies agree that interventions that promote increased physical 
activity improve IBS gastrointestinal symptoms; as well as different aspects of quality of life, fatigue, depression and long-term anxiety

Key words: Irritable bowel syndrome, gastrointestinal diseases, exercise, physical activity, review.

\section{Introducción}

El síndrome de intestino irritable (SII) es el diagnóstico gastroenterológico más común, y se define como la presencia de dolor o molestia abdominal asociada a hábitos intestinales alterados, en ausencia de otra enfermedad que cause dichos síntomas (1). Esta patología se caracteriza por la presencia de constipación o diarrea, distensión abdominal y cambio en la apariencia de las heces. Los síntomas pueden agravarse frecuentemente después de las comidas e incluir náuseas, eructos y pirosis (2). La prevalencia global del SII oscila entre el 5\% al $21 \%$, cuya afectación es mayor en mujeres y personas jovénes (3).

Esta enfermedad posee un curso recurrente crónico, se asocia comúnmente con otros trastornos funcionales, y afecta considerablemente la calidad de vida relacionada con salud. Estas propiedades, aunado a su alta prevalencia y síntomas incapacitantes, le confieren un costo substancial a la sociedad, incluyendo tanto costos directos para el sistema de salud e indirectos como los relacionados al ausentismo laboral (5).

El tratamiento del SIl se dirige a los síntomas predominantes experimentados por el paciente y apunta a su fisiopatología, como lo es el tránsito intestinal acelerado y la hipersensibilidad visceral (5). Las guías clínicas NICE (actualizadas en 2017) recomiendan el enfoque terapeútico basado en el esfuerzo personal (self-help, en inglés) por parte del paciente para el manejo efectivo del SII, ya que actualmente son pocos los tratamientos que ofrecen alivio sintomático satisfactorio. Este abordaje abarca la consejería sobre cambios en el estilo de vida, dieta, actividad física y medicamentos enfocados en los síntomas (6).

Los pacientes con SIl pueden mostrar muchos signos de un estado crónico de estrés. Agregado a los síntomas gastroenterológicos, dichos pacientes usualmente experimentan un amplio rango de otros problemas, como dolores no abdominales, alteraciones psicológicas, bajo autoestima, dificultad en el manejo de situaciones de actividades de la vida diaria, parámetros de estrés anormales, etc. (7).

La exacerbación de los síntomas del SII en presencia de estrés emocional sugiere que las intervenciones mente-cuerpo pueden ser beneficiosas (8). El ejercicio físico se ha vuelto un tema de creciente interés, ya que puede disminuir los síntomas somáticos, así como aliviar el estrés y dolor asociado (3). La presente revisión tiene como objetivo describir el papel de la actividad física como tratamiento no farmacológico del SII.

\section{Diagnóstico}

El diagnóstico se basa en los criterios de Roma IV, los cuales señalan que se deben presentar una historia de al menos seis meses de evolución previa al diagnóstico, caracterizada por dolor o molestia abdominal recurrente una vez por semana en los últimos tres meses asociado a 2 o más de los siguientes criterios: mejoría sintomática con la defecación, inicio asociado con cambios en la frecuencia de deposiciones o inicio asociado con cambios en la apariencia / forma de las heces (9).

\section{Condiciones asociadas}

Existe abundante evidencia de que el SII comparte muchas características con otros síndromes como fibromialgia, síndrome de fatiga crónica y otros trastornos somatoformes. Estos síndromes 
con frecuencia son caracterizados por la ausencia de una etiología física o biológica clara y una inconsistencia en pruebas de laboratorio (7). La asociación con síndromes de dolor somático, trastornos funcionales gastrointestinales y trastornos psiquiátricos (depresión mayor, ansiedad, somatización), refuerzan la posibilidad de una patogénesis compartida (1).

\section{Papel del estrés en la fisiopatología}

Anormalidades en la vía del estrés se han relacionado con la alteración en la permeabilidad intestinal y el desencadenamiento de una cascada de activación inmune que resulta en el SII (9). La activación de la respuesta del estrés o de "lucha o huida" corresponde a una reacción adaptativa mediada por el eje simpático-adrenal-medular para asegurar la sobrevivencia del organismo. La respuesta de estrés puede ser desencadenada por varios estímulos ambientales como miedo, agresión, cambios ambientales inesperados, aislamiento social u otras condiciones patológicas.

Se ha evidenciado que la exposición de dichos estresores desencadena respuestas motoras y sensoriales exageradas en pacientes con SII, lo que podría explicar el desarrollo de los síntomas asociados a la enfermedad (10).

La desregulación o la inactivación inapropiada de la respuesta al estrés puede tener consecuencias en la función intestinal normal. De esta manera se altera la motilidad intestinal, el tránsito fecal y aumenta la sensibilidad al dolor por estimulación visceral. A nivel molecular, un estímulo estresante activa el eje hipotálamo-hipófisisadrenal (HHA) al inducir la secreción del factor liberador de corticotropina (CRF). La hormona adrenocorticotropina (ACTH) es secretada subsecuentemente desde la hipófisis anterior a la circulación, lo cual resulta en la secreción de glucocorticoides desde las glándulas adrenales. Sin embargo, los problemas surgen cuando la regulación del eje HHA se altera, ya que la activación crónica del eje HHA puede resultar en niveles de cortisol elevados; lo cual ha sido involucrado en un número de trastornos como depresión, ansiedad y SII (10).

Estudios de imágenes cerebrales evidencian el rol del sistema nervioso central y el eje "cerebrointestino" al demostrar un procesamiento neurológico alterado de los estímulos viscerales en pacientes con SII. El dolor abdominal recurrente en el SII ha sido atribuido a un umbral de dolor visceral bajo. En varios estudios, se ha reportado que pacientes con SII presentan dolor a volúmenes o presiones de distención de asas intestinales más bajas que la población control, lo cual sugiere que la hiperalgesia visceral puede ser causada por diferencias fisiológicas. Se ha propuesto que dicho incremento en la sensibilidad al estímulo visceral en SII es explicado por la tendencia aumentada de reportar dolor, más que una sensibilidad neuro-sensorial elevada. En un estudio restrospectivo que evaluó la contribución del sobre-reporte en SII se documentó que estos pacientes experimentan marcadamente mayor dolor producto de la distensión intestinal, a pesar de que los umbrales sensoriales rectales no se encontraban alterados (12).

\section{Papel de la actividad física}

Los mecanismos detrás de la asociación entre la actividad física y el SII son desconocidos, debido a que existe gran variabilidad entre sujetos en cuanto al tránsito intestinal fecal y gaseoso. Sin embargo, se ha descrito que la actividad física puede influenciar favorablemente la plasticidad cerebral al facilitar procesos neurogenerativos, neuroadaptativos y neuroprotectores(12). Asimismo, la mejora en la capacidad cardiorrespiratoria y el aumento en actividad física habitual se asocia con la disminución en la severidad de síntomas depresivos y un bienestar emocional mayor. ${ }^{13}$ 
Las interacciones cerebro-intestino pueden jugar un rol en la asociación entre la actividad física y los síntomas en el SII. El estrés induce una exageración de la respuesta neuroendocrina y alteraciones perceptuales viscerales, que puede contrarrestarse con la actividad física mediante su efecto positivo sobre la plasticidad cerebral (14).

Una encuesta realizada en el 2001 demostró que mujeres que padecían de SII eran físicamente menos activas que mujeres sanas. Mujeres con SIl físicamente activas reportaron menos fatiga y menos sensación de evacuación incompleta (13). Un estudio prospectivo, aleatorizado y controlado publicado en el 2011, evaluó a 102 personas, referidas a unidades de gastroenterología desde hospitales comunitarios y hospitales universitarios en Västra Götalandsregion (Suecia), que fueron divididas en un grupo que realizaba actividad física programada, y un grupo control de sujetos físicamente inactivos.

Este estudio mostró que el aumento de la actividad física disminuye la severidad de los síntomas en el SIl y mejora algunas de las dimensiones de la calidad de vida en estos pacientes. Además, se demostró que el aumento en la severidad de los síntomas era significativamente menos prevalente en el grupo físicamente activo que en el grupo control. De forma complementaria, se determinó que el riesgo de síntomas incapacitantes era significativamente mayor en pacientes con SII físicamente inactivos. Por tanto, se concluyó que la actividad física debe ser recomendada como tratamiento de primera línea en estos pacientes(13).

En un estudio de seguimiento publicado en el 2015 llevado a cabo en Suecia, se evaluó los efectos a largo plazo de la actividad física en el manejo de SII. Se incluyeron 39 pacientes, con un seguimiento promedio de 5.2 años. Las actividades más frecuentes reportadas fueron caminatas, aeróbicos y ciclismo. Se demostró que una intervención de 12 semanas seguido por un aumento moderado continuo en actividad física genera efectos positivos a largo plazo en síntomas de SII, calidad de vida, ansiedad y depresión. Se evidenció que $54 \%$ de los pacientes presentaron mejoría clínicamente significativa en los síntomas de SII después de una intervención de 12 semanas comparado con un $43 \%$ en un estudio previo(14).

En un estudio transversal realizado en Irán con 4763 adultos, cuya prevalencia de SII fue de $21.5 \%$, se demostró que en comparación con individuos físicamente activos ( $\geq 1$ hora/semana), aquellas personas sedentarias (menos de 1 hora/semana) poseían 1.27 veces mayor probabilidad de sufrir este síndrome. Sin embargo, dicha asociación entre el sedentarismo y el SII se vio atenuada, con resultados no significativos después de ajustarse por edad, sexo, tabaquismo, e historia médica; y adicionalmente tomando en cuenta prácticas relacionadas con la dieta alimentaria y el índice de masa corporal.

Este estudio concluyó que existe una asociación positiva estadísticamente significativa entre el sedentarismo y el SII en mujeres e individuos de peso normal; no así en individuos con sobrepeso u obesidad, lo cual puede deberse a los efectos de la obesidad en la regulación de las hormonas gastrointestinales, tales como el péptido similar al glucagón 1 (GLP-1), en comparación con sujetos de peso normal(12).

Enuna revisión sistemática publicadaen2018, en el cual no se restringieron publicaciones basadas en su región de desarrollo, lenguaje o tipo de estudio, se estudiaron los efectos del ejercicio en SII. Se analizaron un total de 683 pacientes con SII de 14 ensayos controlados, aleatorizados. Se incluyó el yoga, la caminata/actividad física aeróbica, el Tai Ji, el montañismo y el Baduanjin como clases de ejercicio. La práctica de yoga se relacionó con un mejoramiento global significativamente mayor, menos dolor, constipación y náuseas en adultos 
jóvenes (18-26 años) en comparación con su estado previo. Asimismo, se reportó que la mejoría de síntomas intestinales y autonómicos del SII de predominio diarrea fue mayor en comparación con el uso de loperamida. Lo anterior se cree que se debe al aumento de la actividad parasimpática que experimentó el grupo de yoga(3).

Con respecto al papel de la actividad física aeróbica se describió que los programas de entrenamiento con ejercicios de intensidad bajamoderada por 24 semanas poseían funciones moduladoras inmunes y redox. Esta revisión sistemática reafirmó el beneficio de esta terapia al demostrarse mejoría en cuanto a síntomas gastrointestinales, calidad de vida, ansiedad y comorbilidades asociadas a SII, en comparación con las medidas usuales o de continuar con estilo de vida basal en pacientes con SII. Algunos de los estudios incluidos en la revisión mostraron que el ejercicio era tan efectivo como los fármacos o una dieta baja en FODMAP, e incluso podía ser más efectivo que los fármacos para el mejoramiento de los síntomas gastrointestinales del SII(3).

Una revisión sistemática de 6 ensayos aleatorizados controlados $\mathrm{o}$ aleatorizados cruzados, que incluían adolescentes y adultos, analizó el papel de yoga en el SII. Se evidenciaron los efectos beneficiosos del yoga sobre síntomas gastrointestinales, calidad de vida y ansiedad comparado con sujetos sin tratamiento. Lo anterior podría deberse a que la práctica de yoga corrige la subactividad del sistema nervioso parasimpático inducido por estrés. Además, estudios individuales determinaron que el yoga parece ser igual de efectivo como un programa de caminata en el mejoramiento de los efectos reportados por pacientes. En consecuencia, la práctica de yoga parece ser un tratamiento prometedor y seguro para personas con SII, lo que reafirma el ejercicio como una terapia con efecto positivo sobre el SII (2).
Un estudio llevado a cabo en Suecia documentó mediante entrevistas las experiencias de 15 pacientes de edades entre 31-74 años, a finales del 2014 e inicios 2015. De acuerdo a los datos recolectados, los pacientes expresaron un mayor control de sus movimientos intestinales con la realización de actividad física; experimentaron reducción del dolor abdominal al estar físicamente activos, facilitación del paso de gases, cambios en la frecuencia defecatoria y consistencia de las heces. Además, los pacientes experimentaron un bienestar general, que les permitió relajarse y manejar sentimientos negativos. Estos resultados enfatizaron la importancia de tomar en cuenta las experiencias de los pacientes sobre los efectos de la actividad física(15).

\section{Conclusiones}

Dada la información revisada, se puede concluir que las intervenciones que propician el aumento de la actividad física, entre ellos caminatas, ciclismo, aeróbicos, y yoga, mejoran los síntomas gastrointestinales del SII, así como también diferentes aspectos de la calidad de vida, fatiga, depresión y ansiedad a largo plazo. Por lo tanto, el ejercicio debe considerarse como un abordaje terapéutico accesible y efectivo en pacientes con SII. 


\section{Bibliografía}

1. Chey WD, Kurlander J, Eswaran S. Irritable bowel syndrome: A clinical review. JAMA - J Am Med Assoc. 2015;313(9):949-58.

2. Schumann D, Anheyer D, Lauche R, Dobos $\mathrm{G}$, Langhorst J, Cramer H. Effect of Yoga in the Therapy of Irritable Bowel Syndrome: A Systematic Review. Clin Gastroenterol Hepatol. 2016;14(12):1720-31.

3. Zhou C, Zhao E, Li Y, Jia Y, Li F. Exercise therapy of patients with irritable bowel syndrome: $A$ systematic review of randomized controlled trials. Neurogastroenterol Motil. 2019;31(2):1-10. $\iint$

4. Jerndal P, Ringström G, Agerforz P, Karpefors M, Akkermans LM, Bayati A, et al. Gastrointestinal-specific anxiety: An important factor for severity of GI symptoms and quality of life in IBS. Neurogastroenterol Motil. 2010;22(6):646-54.

5. Camilleri M. Management Options for Irritable Bowel Syndrome. Mayo Clin Proc. 2018;93(12):1858-72.

6. Harvey JM, Sibelli A, Chalder T, Everitt $H$, Moss-Morris R, Bishop FL. Desperately seeking a cure: Treatment seeking and appraisal in irritable bowel syndrome. $\mathrm{Br} \mathrm{J}$ Health Psychol. 2018;23(3):561-79.

7. Eriksson EM, Andrén KI, Kurlberg GK, Eriksson HT. Aspects of the non-pharmacological treatment of irritable bowel syndRome. World J Gastroenterol. 2015;21(40):11439-49.

8. Evans S, Cousins L, Tsao JCl, Sternlieb B, Zeltzer LK. Protocol for a randomized controlled study of lyengar yoga for youth with irritable bowel syndrome. Trials. 2011;12:1-19.

9. Rawla P, Sunkara T, Raj JP. Updated review of current pharmacological and non-pharmacological management of irritable bowel syndrome. Life Sci. 2018;212(September):176-81.

10. O'malley D, Quigley EMM, Dinan TG, Cryan JF. Do interactions between stress and immune responses lead to symptom exacerbations in irritable bowel syndrome? Brain Behav Immun.
2011;25(7):1333-41.

11. Elsenbruch $\mathrm{S}$. Abdominal pain in Irritable Bowel Syndrome: A review of putative psychological, neural and neuro-immune mechanisms. Brain Behav Immun. 2011;25(3):386-94.

12. Sadeghian M, Sadeghi O, Keshteli AH, Daghaghzadeh $\mathrm{H}$, Esmaillzadeh A, Adibi P. Physical activity in relation to irritable bowel syndrome among Iranian adults. PLoS One. 2018;13(10):1-10.

13. Johannesson E, Simrén M, Strid H, Bajor A, Sadik R. Physical activity improves symptoms in irritable bowel syndrome: A randomized controlled trial. Am J Gastroenterol. 2011;106(5):915-22.

14. Johannesson E. Intervention to increase physical activity in irritable bowel syndrome shows long-term positive effects. World J Gastroenterol. 2015;21(2):600.

15. Johannesson E, Jakobsson Ung E, Sadik R, Ringström G. Experiences of the effects of physical activity in persons with irritable bowel syndrome (IBS): a qualitative content analysis. Scand J Gastroenterol. 2018;53(10-11):1194-200. 